\title{
Maackiain Detoxification Contributes to the Virulence of Nectria haematococca MP VI on Chickpea
}

\author{
Jürg Enkerli, ${ }^{1}$ Garima Bhatt, ${ }^{2}$ and Sarah F. Covert ${ }^{2}$ \\ ${ }^{1}$ Department of Botany, and ${ }^{2}$ Daniel B. Warnell School of Forest Resources, University of Georgia, Athens \\ 30602, U.S.A. \\ Accepted 11 December 1997.
}

\begin{abstract}
Nectria haematococca mating population (MP) VI isolates that contain the MAK1 gene are able to degrade maackiain, a chickpea (Cicer arietinum) phytoalexin, to a less toxic compound. To test the contribution of MAK1 to the virulence of $N$. haematococca MP VI on chickpea, the $M A K 1$ gene was disrupted in a highly virulent $\mathrm{Mak}^{+}$isolate or added to a weakly virulent Mak $^{-}$isolate via transformation. The disruption of $M A K 1$ decreased virulence to a moderate level, while addition of multiple copies of $M A K 1$ increased virulence to either a moderate or a high level. These data demonstrate that maackiain detoxification is a determinant, but not the only determinant, of virulence in $N$. haematococca MP VI isolates capable of causing disease on chickpea. $M A K 1$ is located on a 1.6-Mb conditionally dispensable chromosome. To ascertain if there are additional genes influencing virulence toward chickpea stems on the MAK1 chromosome, the loss of this chromosome was chemically induced in an isolate containing the disrupted MAK1 gene. Loss of the MAK1 chromosome did not reduce virulence toward chickpea stems further, thus indicating that no additional genes for virulence on this part of the host plant are located on the MAK1 chromosome.
\end{abstract}

Additional keywords: Fusarium solani.

The accumulation of phytoalexins, low molecular weight antimicrobial compounds produced in response to pathogen attack or wounding, is a well-studied reaction in plants. Phytoalexins were first described by Müller and Börger (1940) in potato and since then have been found in many other plants (Bailey and Mansfield 1982). They constitute a structurally diverse group of chemicals consisting of phenylpropanoid, terpenoid, and fatty acid derivatives (Ebel 1986). A large body of correlative evidence supports the idea that phytoalexins have an important role in the defense of plants against pathogens such as bacteria, fungi, nematodes, and insects (Sharma and Salunkhe 1991). More direct evidence for the importance of phytoalexins as defense compounds was published by Hain et al. in 1993; the resistance of tobacco (Nicotiana tabacum) to Botrytis cinerea was increased by transforming tobacco with a stilbene synthase that enabled the transformants to synthesize the phytoalexin resveratrol.

Corresponding author: Sarah F. Covert; Telephone: 1-706-542-1205; Fax: 1-706-542-8356; E-mail: Covert@BSCR.UGA.EDU
Several fungal pathogens of plants are able to detoxify phytoalexins to less toxic compounds and numerous studies have demonstrated a link between the ability to detoxify phytoalexins and pathogenicity (Weltring 1992). To date, the importance of phytoalexin detoxification for pathogenicity has been demonstrated most convincingly in studies with Nectria haematococca mating population (MP) VI Berk. \& Broome (Fusarium solani) isolates pathogenic on pea (Pisum sativum). Six genes that encode detoxification of the pea phytoalexin pisatin (PDAl to PDA6) have been identified and cloned from N. haematococca MP VI (Weltring et al. 1988; VanEtten et al. 1989; Reimmann and VanEtten 1994). Gene disruption experiments targeting the $P D A 1$ gene revealed that the ability to detoxify pisatin contributed to the virulence of $N$. haematococca MP VI on pea (Wasmann and VanEtten 1996). Furthermore, when a $\mathrm{Pda}^{-}$isolate of $N$. haematococca MP VI was transformed with a highly expressed $P D A$ gene, the virulence of about a third of the transformants was significantly higher than that of the recipient isolate (Ciuffetti and VanEtten 1996). Additional evidence for the importance of pisatin detoxification came from experiments in which two pathogens that are not normally pathogenic on pea were transformed with a highly expressed PDA gene. Both Cochliobolus heterostrophus, a pathogen on maize, and Ascochyta rabiei, a pathogen on chickpea, were able to cause more necrosis on pea when they contained a highly expressed PDA gene (Schäfer et al. 1989; Barz and Welle 1992).

Recent experiments have shown that multiple genes contributing to $N$. haematococca MP VI virulence on pea are located on the PDAl chromosome (Wasmann and VanEtten 1996). PDAl, as well as all the other known genes encoding phytoalexin detoxification in N. haematococca MP VI, are located on conditionally dispensable chromosomes (Miao et al. 1991; Miao and VanEtten 1992a; VanEtten et al. 1994). Such chromosomes are not required for normal saprophytic growth, but contain genes that contribute to growth on specific host plants. For example, during experiments designed to disrupt the $P D A 1$ gene, it was discovered that transformationmediated loss of the entire $P D A 1$ chromosome led to a greater loss of virulence on pea than did simple loss of $P D A 1$ function (Wasmann and VanEtten 1996). In addition, Kistler et al. (1996) showed that mutants lacking $100 \mathrm{~kb}$ of the PDAl chromosome are less virulent than PDAl-disruption strains. These results supported the earlier proposal that conditionally dispensable chromosomes in fungi serve as reservoirs for genes involved in pathogenicity or host specificity in a man- 
ner similar to that of plasmids, which allow bacteria to colonize different habitats (VanEtten et al. 1994).

The goal of this study was to further investigate the significance of phytoalexin detoxification as a virulence determinant in fungi, as well as the role of dispensable chromosomes as carriers of pathogenicity genes. These issues were studied in the context of the interaction between $N$. haematococca MP VI and chickpea (Cicer arietinum). Previous work found that when N. haematococca MP VI field isolates were tested for virulence on chickpea and for their ability to detoxify the chickpea phytoalexin maackiain, all highly virulent isolates were able to detoxify maackiain ( $\mathrm{Mak}^{+}$phenotype), but several $\mathrm{Mak}^{+}$isolates were low in virulence (Lucy et al. 1988). Therefore, the ability to detoxify maackiain appeared to be necessary, but not sufficient, for high virulence of $N$. haematococca MP VI on chickpea. Four genes (MAK1 to MAK4) that encode proteins capable of detoxifying maackiain have been identified in isolates of $N$. haematococca MP VI (Miao and VanEtten 1992a; Covert et al. 1996). In genetic studies, $M A K 1$ and $M A K 2$ were associated with high virulence on chickpea (Miao and VanEtten 1992b). MAKl was recently cloned; it encodes a putative flavin adenine dinucleotide (FAD)-containing mono-oxygenase that metabolizes maackiain to the less toxic compound 1a-hydroxy-maackiain (Covert et al. 1996). Earlier work suggests, therefore, that $M A K 1$ is linked to genes influencing virulence on chickpea, but that the ability to detoxify maackiain is not sufficient for high virulence on chickpea. Herein we address the following question: is $M A K 1$ truly a virulence gene, or is it only linked to an unidentified gene that contributes to virulence on chickpea? Also, because the PDAl chromosome is known to contain more than one gene involved in causing disease on pea, we investigated the possibility that the MAK1 chromosome contains multiple genes associated with virulence on chickpea stems.

\section{RESULTS}

\section{Transformation-mediated gene disruption and gene addition.}

The contribution of MAKI to the virulence of $N$. haematococca MP VI on chickpea was investigated by employing two different approaches. In the first approach the $M A K 1$ gene was disrupted in a highly virulent $\mathrm{Mak}^{+}$isolate. In the second approach the $M A K 1$ gene was added to a weakly virulent $\mathrm{Mak}^{-}$ isolate. In both cases, resistance to hygromycin B was used as the selectable marker. Figure 1 illustrates the construction of the transformation vectors. For the gene disruption experiments, a 379-bp fragment of the MAK1 coding region, which includes a putative FAD-binding domain (Covert et al. 1996), was replaced with a hygromycin B resistance cassette (Fig. 1A). To maximize the amount of genomic DNA flanking the hygromycin B resistance cassette and thus the likelihood of a double cross-over at the MAK1 locus, the disrupted copy of $M A K 1$ was moved into pM1-2 to make pJE6C (Fig. 1A). The $N$. haematococca MP VI genomic DNA flanking the hygromycin B resistance cassette in pJE6C is $1.2 \mathrm{~kb}$ long at the $5^{\prime}$ end and $6 \mathrm{~kb}$ long at the $3^{\prime}$ end. For the $M A K 1$ gene addition experiments, pJE7A was constructed by inserting the hygromycin B resistance cassette into a plasmid containing the intact $M A K 1$ gene (Fig. 1B).
A

\section{Construction of pJE6A}

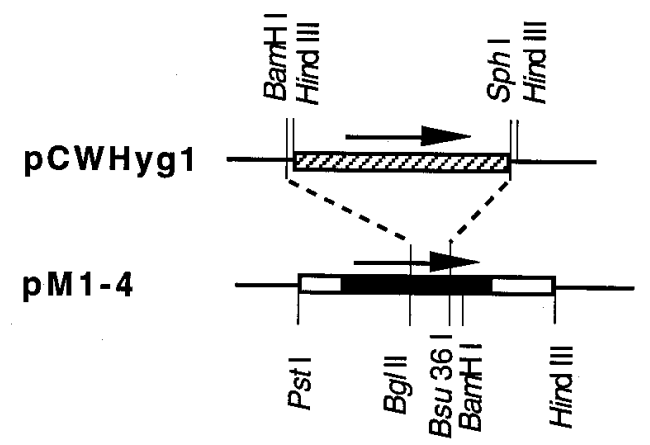

\section{Construction of pJE6C}

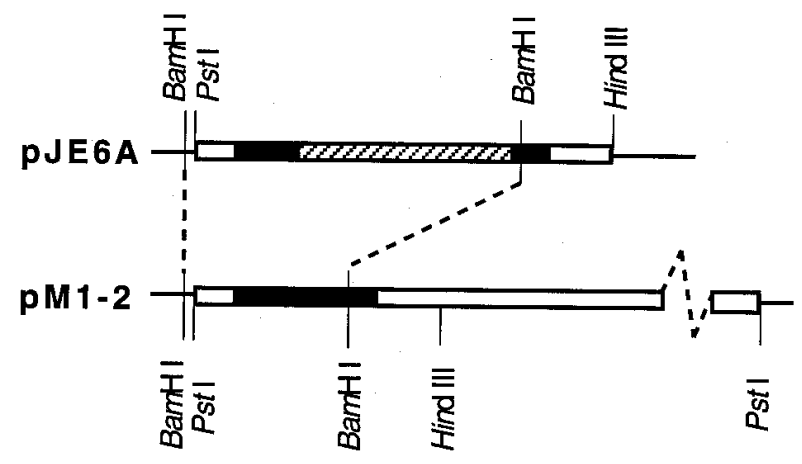

B

\section{Construction of pJE7A}

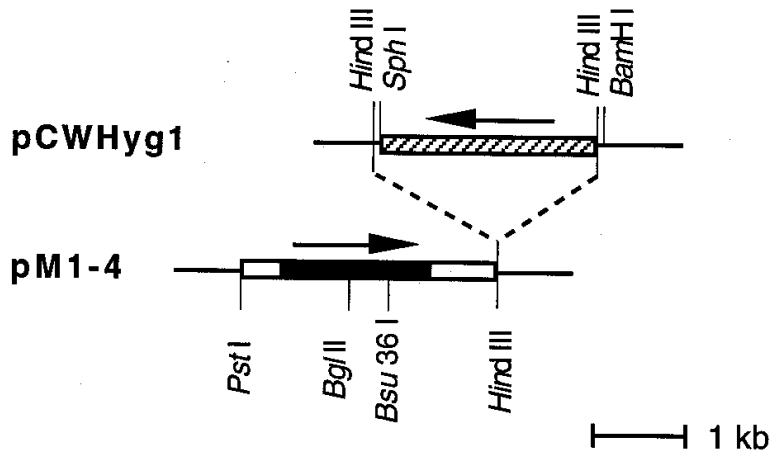

Fig. 1. Construction of $M A K 1$ disruption vector pJE6C and $M A K 1$ addition vector pJE7A. Black boxes represent $M A K 1$ coding sequence; open boxes represent genomic Nectria haematococca MP VI DNA flanking the coding sequence of $M A K 1$; hatched boxes represent the hygromycin $\mathrm{B}$ resistance cassette; horizontal lines represent vector sequences. Arrows indicate direction of transcription. Bar indicating size is shown at bottom. A, Construction of pJE6C. Hygromycin B resistance cassette was isolated from clone pCWHyg1 and inserted into the BglII and Bsu36I site of clone pM1-4 to form the MAK1 disrupted clone pJE6A. $B a m \mathrm{HI}$ fragment of pJE6A containing disrupted MAKl gene was moved into corresponding sites in clone pM1-2 to form clone pJE6C. B, Construction of pJE7A. HindIII fragment containing hygromycin B resistance cassette was isolated from pCWHyg1 and inserted into the HindIII site in pM1-4. 
Thirty-seven hygromycin B-resistant transformants were obtained when the $\mathrm{Mak}^{+}$isolate 230-30-6 (Table 1) was transformed with the MAK1 disruption vector pJE6C. To identify transformants with a "knocked-out" copy of MAK1, all 37 transformants were tested for their ability to detoxify maackiain to 1a-hydroxy maackiain. As demonstrated in Figure 2, four transformants (230KO-1 to $230 \mathrm{KO}-4)$ lost the ability to detoxify maackiain. These four transformants were analyzed further by Southern blot analysis. Transformants 230KO-2 and 230KO-4 appeared to contain a single integration of pJE6C at the $M A K 1$ locus. The verification of correct integration at the $5^{\prime}$ end is shown in Figure 3. The correct integration of pJE6C at the $3^{\prime}$ end was also verified (data not shown). No changes in the karyotype were detected when chromosome-sized DNA of 230KO-2 and 230KO-4 was separated by contour-clamped homogeneous electric field (CHEF) electrophoresis (data not shown). Because 230KO-2 and 230KO-4 appeared to contain mutations only at the MAK1 locus, they were studied further in virulence assays (see below). The other two $\mathrm{Mak}^{-}$transformants, 230KO-1 and 230KO-3, were not analyzed further because of the ectopic pJE6C integration events detected in them (Fig. 3). Four Mak ${ }^{+}$transformants with multiple, ectopic integrations of pJE6C (230TC-1 to 230TC-4) were chosen as controls in subsequent experiments for the effect of transformation on the virulence of 230-30-6 derivatives.

The $\mathrm{Mak}^{-}$isolate 272-26-1 (Table 1) was transformed with the $M A K 1$ addition vector pJE7A. Five transformants (272AD-1 to 272AD-5) were tested for maackiain detoxifying activity. All five transformants were $\mathrm{Mak}^{+}$(Fig. 2). Southern blot analysis revealed that transformant $272 \mathrm{AD}-3$ received two to three copies of pJE7A and the four other transformants received five to 10 copies of pJE7A (data not shown). To create a control for the effects of transformation on virulence, isolate 272-26-1 was transformed with pJE6A. One resulting transformant that remained $\mathrm{Mak}^{-}$and contains multiple ectopic integrations of pJE6A (272TC-1) served as a transformation control for 272-261 derivatives in the following experiments.

\section{Growth rate and sensitivity of transformants to maackiain.}

The growth rate of 230-30-6, 272-26-1, and the transformants derived from them was determined by measuring radial growth of each isolate after 7 days on peptone-glucose agar medium (PGA). The growth rate measurements are summarized in Table 2. All isolates had the same growth rate on PGA. To determine the sensitivity of each isolate toward maackiain, the growth rate on PGA $+50 \mu \mathrm{g}$ of maackiain per $\mathrm{ml}$ was compared with the growth rate on PGA without maackiain. Disruption of the MAK1 gene in isolate 230-30-6 resulted in a 20 to $24 \%$ inhibition of growth by maackiain (Table 2). The sensitivity of the two MAK1-disrupted isolates corresponds to the sensitivity of the $\mathrm{Mak}^{-}$wild-type isolate 272-26-1 and the $\mathrm{Mak}^{-}$transformation control, isolate 272TC1. Adding MAKI copies to the $\mathrm{Mak}^{-}$isolate 272-26-1 produced isolates whose growth is not inhibited by maackiain (Table 2). The data from the transformation controls indicate that transformation alone did not significantly affect sensitivity to maackiain. All transformants were indistinguishable from wild-type isolates in terms of their mycelial color and spore shape (data not shown).

\section{Virulence of Mak $^{-}$transformants.}

To test the effect of MAKI disruption on $N$. haematococca MP VI, the virulence of different isolates was assessed by

Table 1. Isolates used in this study

\begin{tabular}{|c|c|c|}
\hline Origin/isolate(s) & Relevant characteristics $^{\mathbf{a}}$ & Reference(s) \\
\hline \multicolumn{3}{|c|}{ Progeny of lab cross \#230 } \\
\hline $230-30-6$ & MAKl, $\mathrm{Hyg}^{\mathrm{s}}$, has $1.6 \mathrm{Mb}$ chromosome, high virulence on chickpea & Miao and VanEtten 1992a, 1992b \\
\hline \multicolumn{3}{|c|}{ 230-30-6 transformed with pJE6C } \\
\hline $230 \mathrm{KO}-1$ & $\mathrm{Mak}^{-}, \mathrm{Hyg}^{\mathrm{r}}$ & This study \\
\hline $230 \mathrm{KO}-2$ & $\mathrm{Mak}^{-}, \mathrm{Hyg}^{\mathrm{r}}$ & This study \\
\hline $230 \mathrm{KO}-3$ & $\mathrm{Mak}^{-}, \mathrm{Hyg}^{\mathrm{r}}$ & This study \\
\hline 230KO-4 & $\mathrm{Mak}^{-}, \mathrm{Hyg}^{\mathrm{r}}$ & This study \\
\hline 230TC-1 & $M A K 1, \mathrm{Hyg}^{\mathrm{r}}$ & This study \\
\hline 230TC-2 & $M A K 1, \mathrm{Hyg}^{\mathrm{r}}$ & This study \\
\hline 230TC-3 & $M A K 1, \mathrm{Hyg}^{\mathrm{r}}$ & This study \\
\hline 230TC-4 & $M A K 1, \mathrm{Hyg}^{\mathrm{r}}$ & This study \\
\hline \multicolumn{3}{|c|}{$230 \mathrm{KO}-2+25 \mu \mathrm{g}$ of benomyl per $\mathrm{ml}$} \\
\hline 230CL-1 & $\mathrm{Mak}^{-}, \mathrm{Hyg}^{\mathrm{s}}$, lost $1.6 \mathrm{Mb}$ chromosome & VanEtten et al., in press \\
\hline 230CL-2 & $\mathrm{Mak}^{-}, \mathrm{Hyg}^{\mathrm{s}}$, lost $1.6 \mathrm{Mb}$ chromosome & VanEtten et al., in press \\
\hline 230CL-3 & $\mathrm{Mak}^{-}, \mathrm{Hyg}^{\mathrm{s}}$, lost $1.6 \mathrm{Mb}$ chromosome & VanEtten et al., in press \\
\hline 230CL-4 & $\mathrm{Mak}^{-}, \mathrm{Hyg}^{\mathrm{s}}$, lost $1.6 \mathrm{Mb}$ chromosome & VanEtten et al., in press \\
\hline \multicolumn{3}{|c|}{$230 \mathrm{KO}-2+37.5 \mu \mathrm{g}$ of benomyl per $\mathrm{ml}$} \\
\hline 230CL-5 & $\mathrm{Mak}^{-}, \mathrm{Hyg}^{\mathrm{s}}$, lost $1.6 \mathrm{Mb}$ chromosome & VanEtten et al., in press \\
\hline \multicolumn{3}{|c|}{ Progeny of lab cross \#272 } \\
\hline $272-26-1$ & $\mathrm{Mak}^{-}, \mathrm{Hyg}^{\mathrm{s}}$, no $1.6 \mathrm{Mb}$ chromosome, low virulence on chic kpea & Miao et al. 1991; Miao and VanEtten 1992a \\
\hline \multicolumn{3}{|c|}{ 272-26-1 transformed with pJE7A } \\
\hline 272AD-1 & $M A K 1, \mathrm{Hyg}^{\mathrm{r}}$ & This study \\
\hline 272AD-2 & $M A K 1, \mathrm{Hyg}^{\mathrm{r}}$ & This study \\
\hline $272 \mathrm{AD}-3$ & $M A K 1, \mathrm{Hyg}^{\mathrm{r}}$ & This study \\
\hline 272AD-4 & $M A K 1, \mathrm{Hyg}^{\mathrm{r}}$ & This study \\
\hline 272AD-5 & $M A K 1, \mathrm{Hyg}^{\mathrm{r}}$ & This study \\
\hline \multicolumn{3}{|c|}{ 272-26-1 transformed with pJE6A } \\
\hline $272 \mathrm{TC}-1$ & $\mathrm{Mak}^{-}, \mathrm{Hyg}^{\mathrm{r}}$ & This study \\
\hline
\end{tabular}

${ }^{\mathrm{a}} \mathrm{Hyg}^{\mathrm{s}}$ = hygromycin $\mathrm{B}$ sensitive; $\mathrm{Hyg}^{\mathrm{r}}=$ hygromycin $\mathrm{B}$ resistant. 
measuring the lesion length produced by each on chickpea stems. The disruption of MAK1 in isolate 230-30-6 reduced the average length of the lesions produced from $13.2 \mathrm{~mm}$ to 9.2 to $9.8 \mathrm{~mm}$ (Fig. 4). The difference between the virulence of 230-30-6 and both MAK1-disrupted transformants (230KO2 and 230KO-4) was statistically significant (one-tailed $t=6.0$ and 7.3, respectively, $P<0.001$ ) and the magnitude of the effect was large (Cohen's $d=1.1$ and 1.2, respectively). Isolates 230KO-2 and 230KO-4 do not differ significantly in their virulence (two-tailed $t=0.8, P>0.1$ ). The average value of the pooled virulence data from the four $\mathrm{Mak}^{+}$transformation controls (230TC isolates) was not significantly different from that of 230-30-6 (two-tailed $t=1.6, P>0.1$ ) and the effect of transformation was small (Cohen's $d=0.2$ ). When compared individually with 230-30-6, none of the 230TC isolates was significantly different from $230-30-6$ in virulence (data not shown).

The effect of MAK1 disruption on virulence on chickpea was also tested in pot assays by assessing the number of surviving plants 3 weeks after inoculation. Figure 5 shows representative pot assays in which plants were inoculated with 23030-6, 230KO-2, and 272-26-1. The results of all pot assays are summarized in Table 3. Relative to pots in which the plants were infected with 230-30-6, an average of 3.5 more plants survived in pots infected with either $230 \mathrm{KO}-2$ or $230 \mathrm{KO}-4$. This difference in the number of surviving plants is statistically significant (one-tailed $t=4.1$ and $2.4, P<0.05$ ) and the magnitude of the effect is large with each transformant (Cohen's $d=2.9$ and 3.3). The size of the surviving plants infected with isolates $230 \mathrm{KO}-2$ and $230 \mathrm{KO}-4$ varied between different pots. In some experiments none of the surviving plants was taller than $4.5 \mathrm{~cm}$, in other pots all surviving plants were taller than $12 \mathrm{~cm}$ (data not shown).

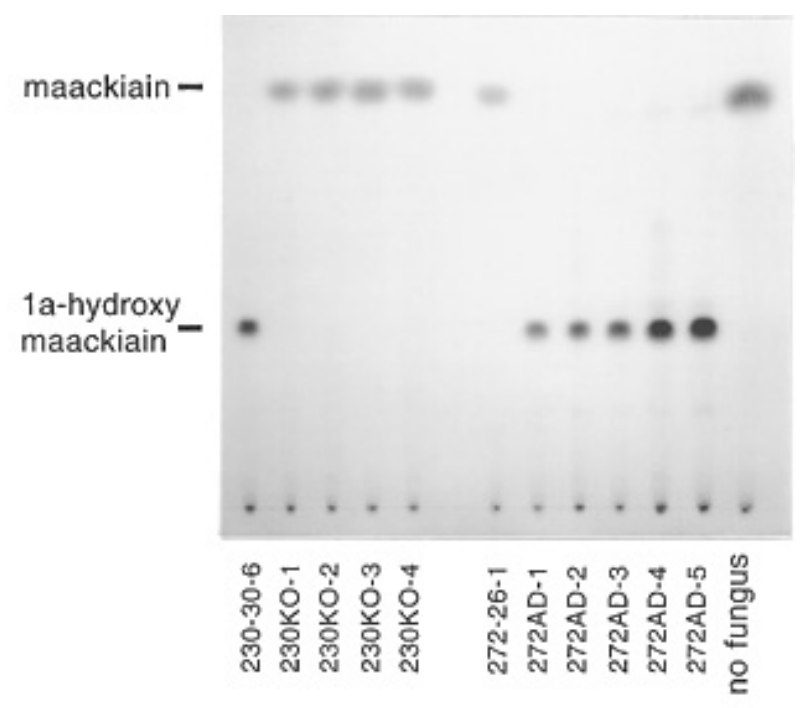

N. haematococca MPVI isolates

Fig. 2. Thin-layer chromatography plate demonstrating maackiainmetabolizing activity of wild-type isolates 230-30-6 (MAK1) and 27226-1 ( $\left.\mathrm{Mak}^{-}\right), \mathrm{Mak}^{-}$transformants of 230-30-6 designated 230KO-1, -2, -3 , and -4 , and $\mathrm{Mak}^{+}$transformants of 272-26-1 designated 272AD-1, -2, $-3,-4$, and -5 . The "no fungus" lane contained maackiain that was not exposed to any isolate. Mobilities of maackiain and 1a-hydroxy maackiain are indicated on left.

\section{Virulence of $\mathrm{Mak}^{+}$transformants.}

Adding the MAK1 gene to isolate 272-26-1 increased the virulence of all five 272AD transformants; however, the amount of increase varied widely from 4.8 to $10.4 \mathrm{~mm}$ (Fig. 6). The difference between the virulence of 272-26-1 and each of the five $M A K 1$-added transformants (272AD isolates) was statistically significant (one-tailed $t=6.9$ to $17.9, P<0.001$ ) and the magnitude of the effect was very large in each case (Cohen's $d=$ 3.3 to 7.1). Transforming 272-26-1 with pJE6A increased average lesion length by $1.3 \mathrm{~mm}$ (Fig. 6). This was a statistically significant difference (two-tailed $t=3.6, P<0.001$ ), and the effect was large (Cohen's $d=0.9$ ). Therefore, when the effect of transformation is considered, the net increase in lesion length due to $M A K 1$ addition ranged from 3.5 to $9.1 \mathrm{~mm}$.
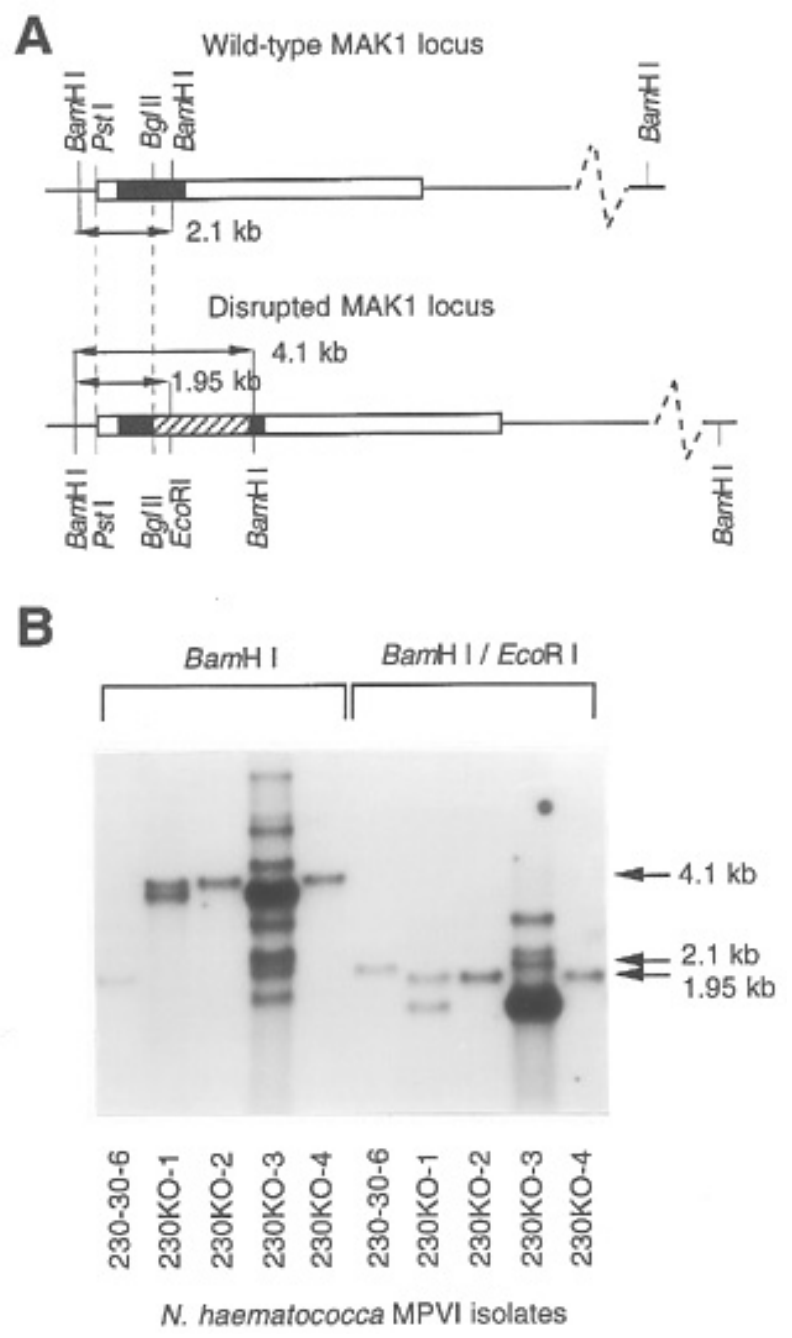

Fig. 3. Southern blot analysis of isolates that lost maackiainmetabolizing activity. A, Partial restriction map of wild-type and disrupted MAK1 locus. DNA present on the transformation vector is boxed. Black boxes represent MAK1 coding sequence; hatched box represents hygromycin B resistance cassette. Vertical dashed lines indicate the PstI$B g I I I$ fragment used as probe in $\mathbf{B}$. Sizes of fragments expected to hybridize to the probe are indicated. B, Southern blot of 230-30-6 (MAKI) and $\mathrm{Mak}^{-}$transformants of 230-30-6 probed with the PstI-BgIII fragment of the MAK1 coding sequence. Brackets on top indicate lanes containing DNA digested with BamHI or with BamHI and EcoRI. Each lane contains $5 \mu \mathrm{g}$ of DNA except the BamHI digest of 230-30-6, which contains $2 \mu \mathrm{g}$ of DNA. 
Induced loss of the 1.6-Mb dispensable

MAK1 chromosome.

To determine if there are additional genes influencing virulence toward chickpea stems on the $1.6-\mathrm{Mb}$ chromosome in isolate 230-30-6, loss of the chromosome was induced chemically and the effect on virulence measured in stem lesion assays. The $M A K 1$ chromosome was tagged with the hygromycin B resistance marker when the MAKl gene was disrupted in isolates $230 \mathrm{KO}-2$ and $230 \mathrm{KO}-4$. This made it possible to screen for loss of the dispensable chromosome by screening for loss of hygromycin B resistance. Isolate 230KO-2 was grown in liquid culture in the presence of benomyl, a fungicide that disrupts the spindle apparatus during mitosis. Following the benomyl treatment, approximately 3,600 spores were screened for the loss of hygromycin B resistance. Twelve hygromycin B-sensitive isolates were identified, and five of them (isolates 230CL-1 to 230CL-5) were analyzed further. All five isolates appeared to have lost the 1.6-Mb chromosome when examined by CHEF electrophoresis (VanEtten et al., in press) and none of the other chromosomes appeared altered in size (data not shown). To confirm that no translocation events were involved in the apparent loss of the chromosome, CHEF-gel separated chromosomal DNA from the five 230CL isolates and from isolate $230 \mathrm{KO}-2$ was probed with the isolated $M A K 1$ chromosome. The probe hybridized weakly to several chromosomal bands in all isolates on the blot and very strongly to the MAK1 chromosomal band in isolate $230 \mathrm{KO}-2$ (data not shown). The weak hybridization signals presumably were due to the presence of repeated DNA sequences, such as centromeres, telomeres, and Nht1, a putative transposable element that is present on multiple chromosomes in 230-30-6 (Enkerli et al. 1997; J. Enkerli, unpublished). As a complementary approach, a Southern blot of EcoRI-digested genomic DNA of each 230CL isolate was probed with a collection of 52 cosmid clones that are distributed along the chromosome. This clone collection is a subset of the 66 clones composing the minimal map of the 1.6-Mb MAK1 chromosome (Enkerli

Table 2. Growth of Nectria haematococca MP VI wild-type isolates and transformants in the absence and presence of maackiain

\begin{tabular}{lcccc}
\hline Isolate & Mak $^{\mathbf{a}}$ & $\begin{array}{c}\text { Growth } \\
- \text { maackiain }^{\mathbf{b}} \\
(\mathbf{c m})\end{array}$ & $\begin{array}{c}\text { Growth } \\
\text { maackiain }^{\mathbf{b}} \\
(\mathbf{c m})\end{array}$ & $\begin{array}{c}\text { Inhibition by }_{\text {maackiain }^{\mathbf{c}}} \\
(\mathbf{\%})\end{array}$ \\
\hline 230-30-6 & + & $2.5 \pm 0.0$ & $2.5 \pm 0.1$ & 0 \\
230KO-2 & - & $2.5 \pm 0.0$ & $2.0 \pm 0.0$ & 20 \\
230KO-4 & - & $2.5 \pm 0.1$ & $1.9 \pm 0.0$ & 24 \\
230TC-1 & + & $2.5 \pm 0.1$ & $2.5 \pm 0.1$ & 0 \\
230TC-2 & + & $2.5 \pm 0.0$ & $2.4 \pm 0.0$ & 4 \\
230TC-3 & + & $2.5 \pm 0.1$ & $2.4 \pm 0.0$ & 4 \\
230TC-4 & + & $2.3 \pm 0.0$ & $2.3 \pm 0.0$ & 0 \\
272-26-1 & - & $2.5 \pm 0.1$ & $1.9 \pm 0.0$ & 24 \\
272AD-1 & + & $2.5 \pm 0.0$ & $2.5 \pm 0.0$ & 0 \\
272AD-2 & + & $2.5 \pm 0.0$ & $2.4 \pm 0.0$ & 4 \\
272AD-3 & + & $2.5 \pm 0.0$ & $2.5 \pm 0.0$ & 0 \\
272AD-4 & + & $2.4 \pm 0.0$ & $2.4 \pm 0.0$ & 0 \\
272AD-5 & + & $2.5 \pm 0.0$ & $2.5 \pm 0.0$ & 0 \\
272TC-1 & - & $2.5 \pm 0.1$ & $1.9 \pm 0.0$ & 24 \\
\hline
\end{tabular}

${ }^{a}$ Ability to detoxify maackiain.

${ }^{\mathrm{b}}$ Radial growth of mycelial colonies after 7 days on peptone glucose agar in absence (-) or presence (+) of maackiain $(50 \mu \mathrm{g} / \mathrm{ml})$. Average of two experiments \pm standard deviation.

${ }^{c}$ [(growth - maackiain $)-($ growth + maackiain $\left.)\right] /$ (growth - maackiain $)$ $\times 100 \%$.
1997). Clones containing repeated DNA sequences found on other chromosomes were not included in the probe subset in order to prevent cross-hybridization with other chromosomes. This clone collection is estimated to cover $77 \%$ of the MAKI chromosome (Enkerli 1997). The result of this experiment is shown in Figure 7. Numerous fragments in isolates 230-30-6 and $230 \mathrm{KO}-2$ hybridized to the probe representing the MAKI chromosome, whereas 230CL isolates that appeared to have lost the chromosome (based on CHEF analysis) did not hybridize to the probe. When tested in a radial growth experiment on PGA plates, the growth rate of the 230CL isolates was identical to the growth rate of isolate 230KO-2 (VanEtten et al., in press). Stem assays were carried out to determine the effect of $M A K 1$ chromosome loss on the virulence of $N$. haematococca MP VI. Figure 8 summarizes the virulence data of the isolates that lost the 1.6-Mb dispensable chromosome. The virulence of all five 230CL isolates toward chickpea stems was virtually indistinguishable from that of 230KO-2, their progenitor.

\section{DISCUSSION}

The goal of this study was to determine if MAKl contributes to the virulence of $N$. haematococca MP VI on chickpea. Disrupting the $M A K 1$ gene resulted in a significant decrease in average lesion length in the stem assay, and a significant reduction in killing effectiveness in the pot assay. However, in both types of assays, the two MAKl-disrupted isolates were still moderately virulent relative to isolate 272-26-1, suggesting that there are additional genes in 230-30-6 that contribute to the virulence of this fungus on chickpea. We conclude,

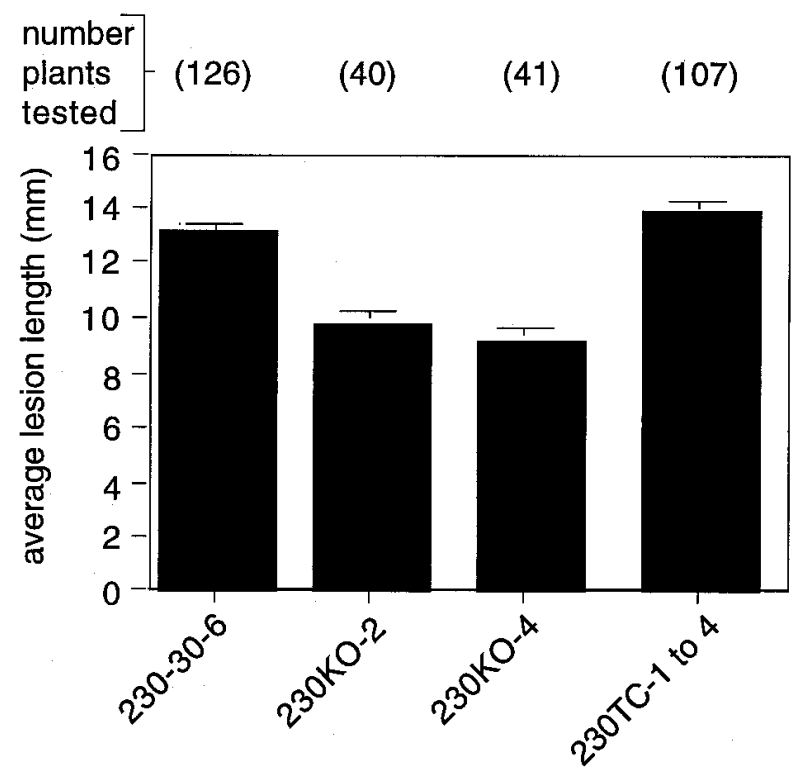

\section{N. haematococca MPVI isolates}

Fig. 4. Virulence on chickpea of 230-30-6 (MAK1), 230KO-2, and 230KO-4 (Mak ${ }^{-}$transformants of 230-30-6) measured as lesion length on inoculated chickpea stems. Lesion length values of the four transformation control isolates (230TC-1 to 230TC-4) were pooled and one average value calculated. Number of plants inoculated with each isolate is indicated in parentheses. Columns represent average lesion length and bars represent standard error. 
therefore, that $M A K 1$ is in fact a determinant of virulence in this host-pathogen interaction, but that it is responsible for only a portion of the disease-causing capacity of isolate 23030-6. This idea agrees well with the results of earlier studies, which suggested that maackiain detoxification is necessary, but not sufficient, for high virulence on chickpea .

In general terms, the effect of $M A K 1$ gene disruption on virulence toward chickpea is similar to the effect of PDAI gene disruption on virulence toward pea (Wasmann and

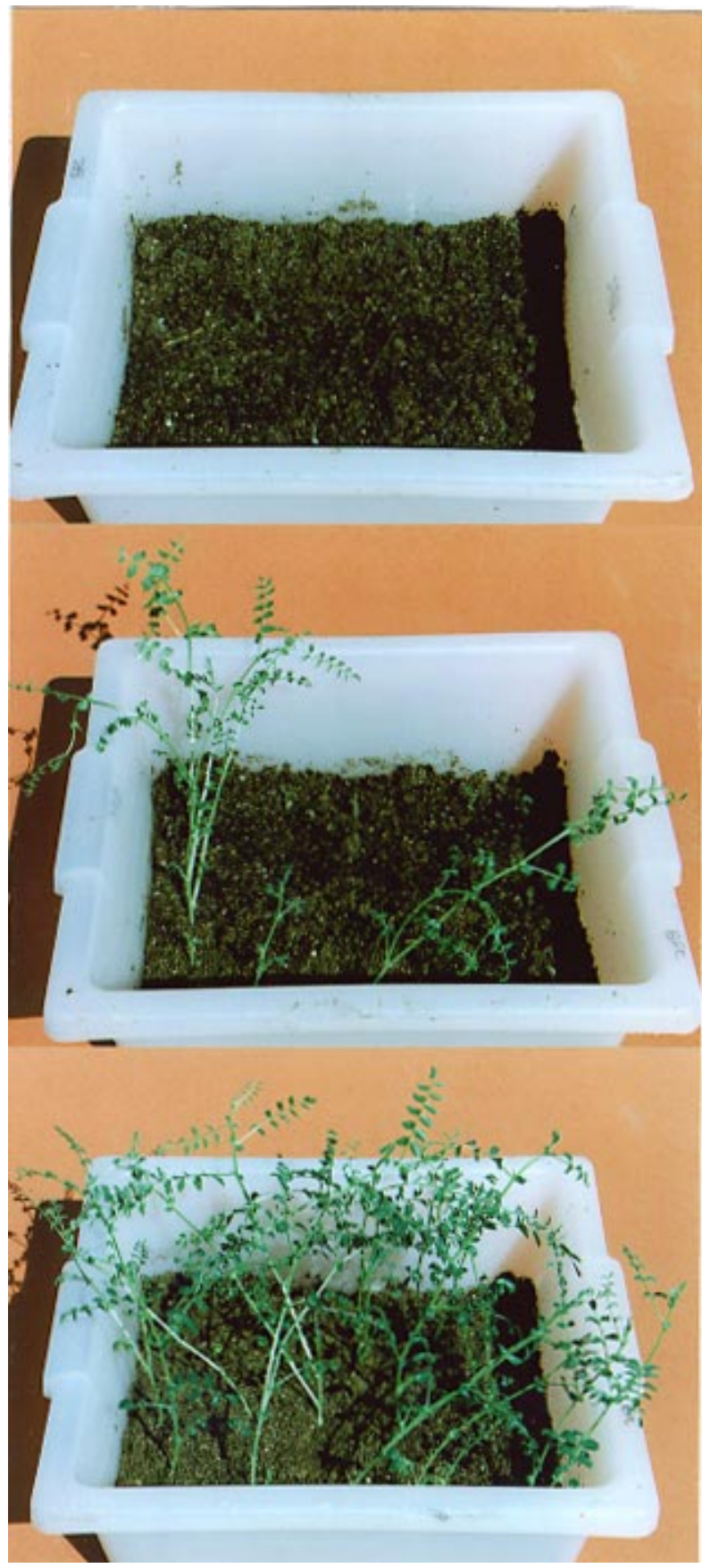

Fig. 5. Representative pot assays of chickpea plants infected with (top) 230-30-6 (MAK1), (middle) 230KO-2 ( $\mathrm{Mak}^{-}$transformant of 230-30-6), and (bottom) 272-26-1 ( $\mathrm{Mak}^{-}$wild type). In each pot, three rows of five germinated seeds were inoculated with one isolate of Nectria haematococca MP VI and incubated for 3 weeks.
VanEtten 1996) in that both mutations caused a partial reduction in N. haematococca MP VI virulence on the corresponding host plant. To allow a more direct comparison of these two data sets, we calculated effect size (Cohen's $d$ ) from the lesion length data presented by Wasmann and VanEtten (1996). In their study, PDAl was disrupted in three different $N$. haematococca MP VI isolates (77-13-4, 77-13-5, and 77-13-7). The average effect size due to disruption of $P D A 1$ varies with each background isolate, i.e., in 77-13-4 $d=1.4$, in 77-13-5 $d=1.7$, and in 77-13-7 $d=1.1$. The difference between the effect sizes associated with 77-13-5 and 77-13-7 raises the possibility that the contribution of pisatin detoxification to virulence on pea is moderately influenced by other loci in the genome. This analysis also makes it clear that the magnitude of the effect attributable to $M A K 1$ disruption ( $d=1.1$ to 1.2 ) is equivalent to the effect due to PDAl disruption in isolate 77-13-7.

The results from the addition of $M A K 1$ to a $\mathrm{Mak}^{-}$isolate lend further support to the idea that $M A K 1$ is an important factor for virulence on chickpea in N. haematococca MP VI. All five transformants that received the $M A K 1$ gene were significantly more virulent than 272-26-1, the $\mathrm{Mak}^{-}$transformation recipient. Even though the transformation control 272TC-1 was significantly more virulent than its background isolate, 272-26-1, the effect of transformation on virulence was relatively small. Because the magnitude of effect size (Cohen's $d$ ) upon addition of MAK1 is 3.7 to 7.9 times larger than effect size due to transformation alone, we conclude that this experiment also indicates that MAK1 has a meaningful effect on the virulence of $N$. haematococca MP VI on chickpea. All five 272AD transformants received multiple copies of the $M A K 1$ gene; therefore, variation in virulence between these isolates may reflect gene dosage and positional effects. Although we did not directly test the impact of multiple copies of $M A K 1$ on virulence, there may be a positive correlation between copy number and virulence as isolate 272AD-3 contained the fewest copies of MAK1 (data not shown) and demonstrated the lowest virulence among the 272AD transformants (Fig. 6).

The effect of $M A K 1$ gene addition on virulence toward chickpea was much more dramatic than the results obtained when $\mathrm{Pda}^{-}$isolates were transformed with a functional PDA gene; only about one-third of the $\mathrm{Pda}^{+}$transformants were moderately more virulent on pea (Ciuffetti and VanEtten 1996). Also, addition of multiple $P D A$ copies to an isolate that already contained a $P D A$ gene did not increase its virulence on pea. As a result, it was concluded that the expression of a single, highly expressed PDA gene provides the optimal level of pisatin detoxification activity (Ciuffetti and VanEtten 1996).

When dispensable chromosomes contain repeated sequences that are also present on other chromosomes in the genome, it is difficult to conclusively demonstrate that apparent loss of a dispensable chromosome is not due to its translocation to another position in the genome. Because the $M A K 1$ chromosome contains several copies of $N h t 1$, a putative transposon that is also present on other chromosomes in the 230-30-6 genome (Enkerli et al. 1997; J. Enkerli, unpublished), two experiments were done to assess if the $230 \mathrm{CL}$ isolates had lost the MAK1 chromosome. Although neither the CHEF blot probed with the isolated MAK1 chromosome (data not shown), nor the genomic Southern 
probed with a collection of clones representing $77 \%$ of the chromosome (Fig. 7) could be unequivocal, the results of both experiments support the conclusion that benomyl exposure induced loss of the $M A K 1$ chromosome and not its translocation. The likelihood that undetected translocation of a piece of the MAKl chromosome is causing maintenance of moderate virulence in the $230 \mathrm{CL}$ isolates is low in light of the fact that all five $230 \mathrm{CL}$ isolates produce chickpea lesions of the same average length. In other words, this virulence result could only have been obtained if all five isolates retained the same undetected piece of the MAK1 chromosome. We conclude, therefore, that loss of the MAKI chromosome did not further reduce the virulence of $N$. haematococca MP VI on chickpea stems because MAK1 is the only gene on the chromosome that contributes to the virulence of 230-30-6 on this part of the host plant. This result contrasts with previous analysis of the PDAl chromosome in which multiple loci conditioning virulence toward pea stems and roots were found to be on the PDAl chromosome (Kistler et al. 1996; Wasmann and VanEtten 1996). The role of the MAK1 chromosome during infection of chickpea roots has not yet been investigated (i.e., tested in pot assays); therefore, it remains possible that genes contributing to infection of roots and/or growth in the chickpea rhizosphere are present on the $M A K 1$ chromosome.

For many years it has been difficult to directly test the suspected function of phytoalexins in disease resistance. Only recently have the critical experiments been done to address this question. It is now clear that enabling a plant to produce a particular phytoalexin increases its resistance toward a pathogen (Hain et al. 1993) and that disrupting pathogen genes encoding phytoalexin detoxification reduces virulence on specific host plants (Wasmann and VanEtten 1996; this study). Furthermore, adding genes encoding phytoalexin detoxification can increase virulence (Ciuffetti and VanEtten 1996; this study) and enable pathogens not normally pathogenic on a particular host plant to cause symptoms (Schäfer et al. 1989; Barz and Welle 1992). These experiments demonstrate that pathogen-induced accumulation of phytoalexins in plants truly is a defense mechanism, provided that the attacking pathogen is not able to overcome this defense reaction by degrading the phytoalexins.

Table 3. Effect of MAK1 disruption on virulence of Nectria haematococca MP VI on chickpea as assessed in a pot assay

\begin{tabular}{|c|c|c|}
\hline Isolate & $\begin{array}{l}\text { Average number } \\
\text { of survivors } \pm \text { SD }^{\mathrm{a}}\end{array}$ & Qualitative comments \\
\hline $230-30-6$ & 0 & Most plants were completely rotten, a few plants had some green tissue left. \\
\hline $230 \mathrm{KO}-2$ & $3.5 \pm 1.7$ & $\begin{array}{l}\text { Thirty-three percent of surviving plants were symptom free except for a lesion at inoculation site ( } 2 \text { to } 5 \mathrm{~mm}) \text {. Sixty- } \\
\text { six percent of surviving plants had lesions on stem and/or leaves. }\end{array}$ \\
\hline $230 \mathrm{KO}-4$ & $3.5 \pm 2.1$ & Same as $230 \mathrm{KO}-2$. \\
\hline $272-26-1$ & $15.0 \pm 0$ & Plants had no symptoms except for a lesion at inoculation site (2 to $5 \mathrm{~mm}$ ). \\
\hline Uninfected & $15.0 \pm 0$ & No symptoms. \\
\hline
\end{tabular}

${ }^{a}$ Survivors were plants that developed like uninfected plants of the same age, except that they sometimes were smaller and sometimes had a few small lesions (up to $5 \mathrm{~mm}$ ) on leaves or stem. Average number of survivors calculated from four independent experiments (15 plants each) for isolates 230 30-6, 230KO-2, and 272-26-1, and two independent experiments (15 plants each) for isolate 230KO-4 and uninfected plants. SD = s tandard deviation.

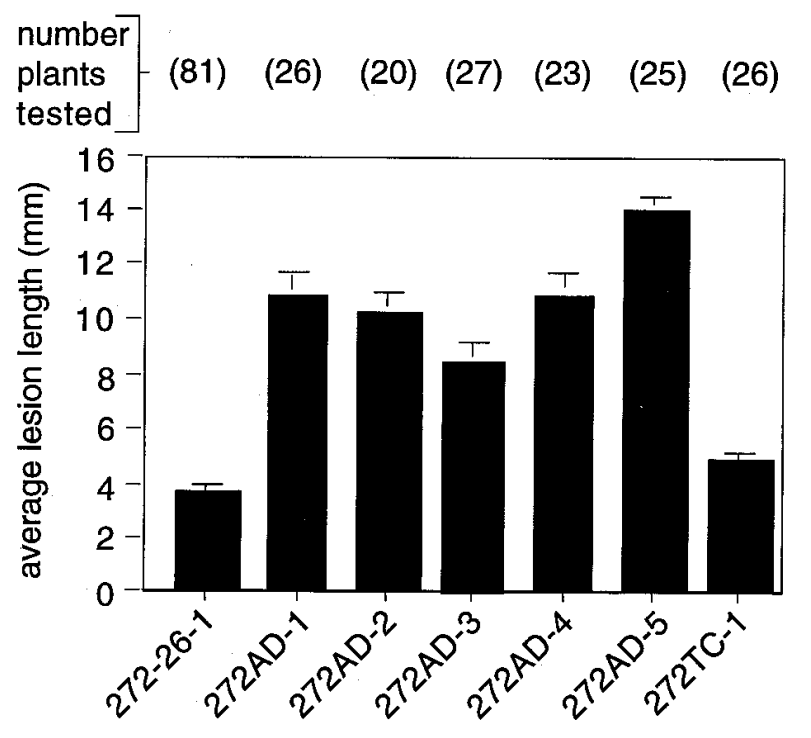

\section{N. haematococca MPVI isolates}

Fig. 6. Virulence on chickpea of 272-26-1 ( $\left.\mathrm{Mak}^{-}\right), 272 \mathrm{AD}-1,-2,-3,-4$,

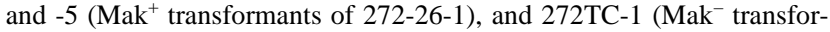
mant of 272-26-1) measured as lesion length on inoculated chickpea stems. Number of plants inoculated with each isolate is indicated in parenthesis. Columns represent average lesion length and bars represent standard error.

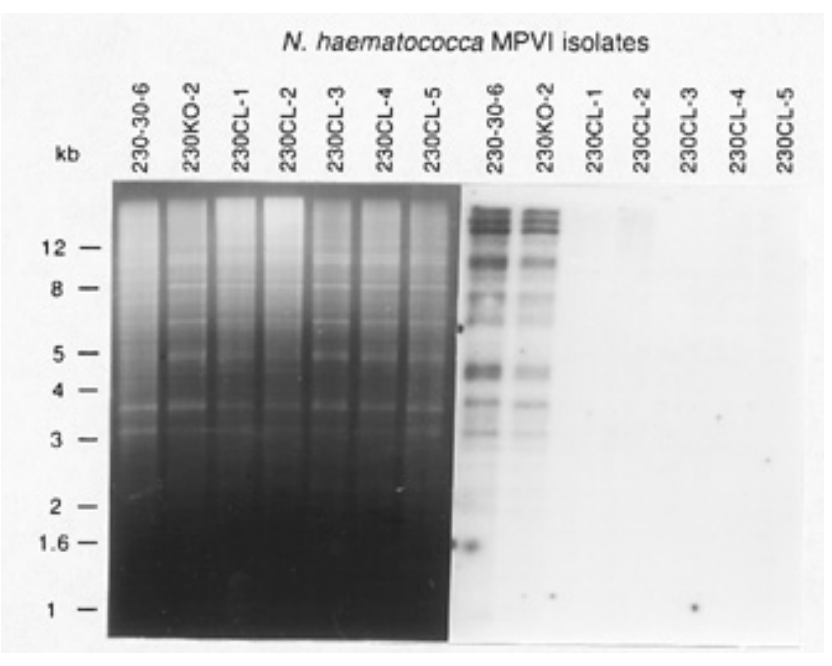

Fig. 7. Southern blot analysis of 230-30-6 (MAK1), 230KO-2 (Mak transformant of 230-30-6), and five strains derived from 230KO-2 after benomyl treatment (230CL-1, -2, -3, -4, and -5). Genomic DNA from each isolate was digested with EcoRI, separated on a $0.8 \%$ agarose gel, and Southern blotted. Corresponding Southern blot was probed with a cosmid collection representing $77 \%$ of the $1.6-\mathrm{Mb}$ dispensable chromosome. 


\section{MATERIALS AND METHODS}

Fungal isolates and growth conditions.

All Nectria haematococca MP VI isolates used in this study, their origins, and their relevant characteristics are listed in Table 1. All isolates were grown and maintained on V8 agar medium (Miller 1955) at $28^{\circ} \mathrm{C}$ in the dark.

\section{DNA manipulations.}

Standard molecular techniques were used throughout (Sambrook et al. 1989). Genomic DNA was isolated as described in Covert et al. (1996). Gel blotting and Southern blot hybridization were performed according to protocols used in Enkerli et al. (1997). DNA for analysis by CHEF electrophoresis was prepared as described in Enkerli et al. (1997) and separated on a CHEF DR III unit (BioRad, Hercules, CA). Conditions for separating the entire karyotype were as follows: $0.8 \%$ agarose in $1 \times$ TAE (40 mM Tris-acetate $\mathrm{pH} 8.5,2$ $\mathrm{mM}$ EDTA), $106^{\circ}$ reorientation angle, $2 \mathrm{~V} / \mathrm{cm}, 1200 \mathrm{~s}$ to 2400 $\mathrm{s}$ switch time, $72 \mathrm{~h}, 14^{\circ} \mathrm{C}$. The probe representing the MAK1 chromosome (Fig. 7) was prepared by mixing equal amounts of 52 cosmids (representing $77 \%$ of the chromosome) and using approximately $500 \mathrm{ng}$ of the mixture for the labeling reaction (DECAprime II DNA labeling Kit, Ambion, Austin, TX).

\section{Construction of transformation vectors.}

The MAK1 gene-disruption vector was prepared by replacing a 379-bp fragment in the $M A K 1$ gene with a hygromycin B resistance cassette consisting of the Aspergillus niger glucoamylase promoter ( $g l a A)$, the hph gene from Escherichia coli, and the Aspergillus nidulans trpC terminator from pCWHyg1 (provided by C. Wasmann and H. VanEtten, University of Arizona). pCWHyg1 was cut with $S p h$ I, blunt-ended

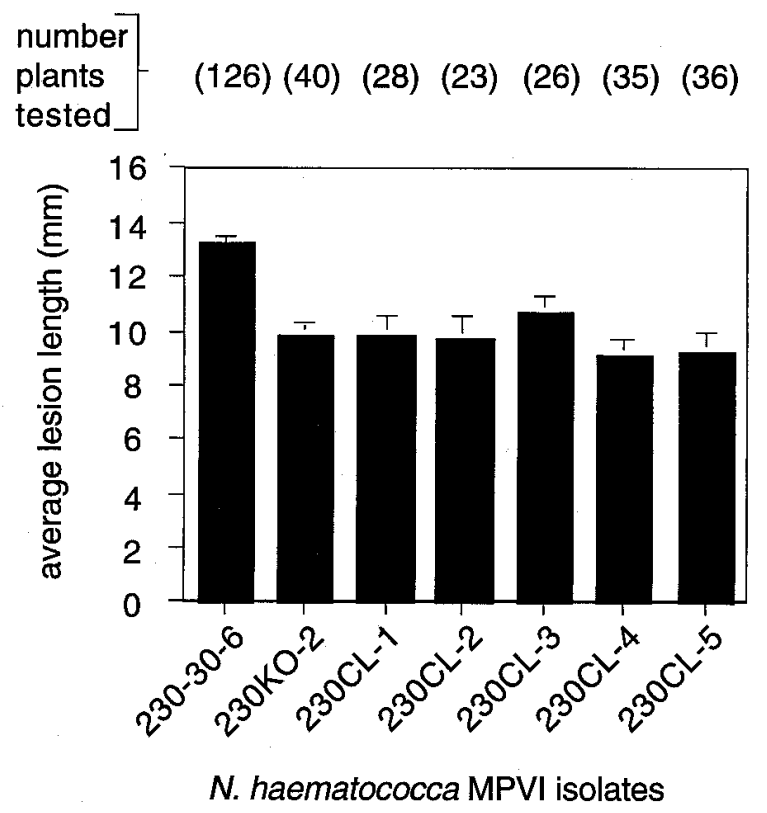

Fig. 8. Virulence on chickpea of 230-30-6 (MAK1), 230KO-2 (Maktransformant of 230-30-6), and 230CL-1, -2, -3, -4, and -5 (derived strains that lack the 1.6-Mb MAK1 chromosome) measured as lesion length on inoculated chickpea stems. Number of plants inoculated with each isolate is indicated in parenthesis. Columns represent average lesion length and bars represent standard error. with T4 DNA polymerase, and then digested with BamHI to isolate the 2.363-kb fragment containing the hygromycin B resistance cassette. This fragment was isolated from an agarose gel. The MAK1 clone pM1-4 (Covert et al. 1996) was digested with Bsu36I, filled in with Klenow to form a blunt end, and then cut with $B g l \mathrm{II}$, which creates a BamHIcompatible overhang. The resulting 5.289-kb fragment was isolated from an agarose gel and ligated to the isolated hygromycin B resistance cassette to create plasmid pJE6A (Fig. 1). pJE6C was constructed by isolating the disrupted MAK1 fragment from pJE6A with a BamHI digest and inserting it into the corresponding dephosphorylated BamHI sites in pM12 as indicated in Figure 1. pM1-2, containing an approximately 9-kb PstI genomic DNA fragment from $N$. haematococca MP VI isolate 156-30-6, was constructed during the original cloning of MAKl (Covert et al. 1996; S. F. Covert, unpublished).

Transformation vector pJE7A was created by inserting the 2.342-kb HindIII fragment from pCWHyg1 (containing the hygromycin B resistance cassette) into the dephosphorylated HindIII site 3' of pM1-4 (Fig. 1). The construct in which the $h p h$ gene and the $M A K l$ gene are transcribed in opposite directions was used.

\section{Fungal transformation.}

Protoplasts for transformation were prepared as described in Enkerli et al. (1997). After washing the protoplasts twice in 10 $\mathrm{ml}$ of SE (1 M sorbitol, $50 \mathrm{mM}$ EDTA, $\mathrm{pH} 8.0$ ), and once in STC (1.2 M sorbitol, $25 \mathrm{mM}$ TRIS-HCl pH 7.0, $25 \mathrm{mM}$ $\mathrm{CaCl}_{2}$ ) the protoplasts were resuspended in STC at a concentration of $1 \times 10^{8}$ per ml. Transformation was carried out as described by Covert et al. (1996) with some modifications in the plating procedure. Each transformation reaction was split into two aliquots of $500 \mu \mathrm{l}$, mixed with $500 \mu \mathrm{l}$ of $20 \%$ polyethylene glycol 4000 and $4 \mathrm{ml}$ of molten $\left(48^{\circ} \mathrm{C}\right)$ regeneration top agar $(1.2 \%$ sorbitol, $0.1 \%$ yeast extract, $0.1 \%$ casein hydrolysate, $1 \%$ agar), and poured onto a $20-\mathrm{ml}$ base layer agar plate $(0.6 \%$ sorbitol, $0.1 \%$ yeast extract, $0.1 \%$ casein hydrolysate, $1 \%$ agar, $62.5 \mu \mathrm{g}$ of hygromycin B per $\mathrm{ml}$ ). Plates were incubated for 5 to 6 days at $28^{\circ} \mathrm{C}$ in the dark. Emerging transformants were cut from the transformation plate and placed on M100 minimal medium plates (Stevens 1974) containing 50 $\mu \mathrm{g}$ of hygromycin B per ml. All transformants were subsequently propagated from single-spore, isolated colonies.

Assays for metabolic activity and sensitivity to maackiain.

The ability to metabolize maackiain was assayed by thinlayer chromatography as described by Miao and VanEtten (1992a) except that maackiain was added at the same time conidia were added to the medium, and the cultures were incubated for only $24 \mathrm{~h}$ before extraction. To test sensitivity to maackiain, the growth rate of different isolates on peptoneglucose agar medium (VanEtten 1973) in the presence or absence of $50 \mu \mathrm{g}$ of maackiain per ml was determined as described by Lucy et al. (1988). The growth of each isolate was measured on two independent plates 7 days after inoculation.

\section{Virulence assays.}

The stem assay as described by Lucy et al. (1988) was used with some modifications. Chickpea seeds (Cicer arietinum, accession no. B-50245; W. Atlee Burpee, Warminster, PA), 
were soaked in water for $5 \mathrm{~min}$, surface sterilized with $5.25 \%$ sodium hypochlorite for $2 \mathrm{~min}$, and rinsed four times with sterile water (15 min each). Single sterilized seeds were planted in test tubes $(2.5 \times 15.0 \mathrm{~cm})$ containing sterile soil (Peat lite mix; C. Fafard, Agawam, MA) and watered with 15 $\mathrm{ml}$ of sterile water. Seeds were covered with $1 \mathrm{~cm}$ of sterile soil. Plants were grown in the dark for 5 days at $27^{\circ} \mathrm{C}$ and then put into an illuminated incubator (Model 818; Precision Scientific, Chicago, IL) for $16 \mathrm{~h}$ at $27^{\circ} \mathrm{C}$. The inoculum was prepared as described by Lucy et al. (1988). Emerging plants ( 2 to $5 \mathrm{~cm}$ long) were inoculated $1 \mathrm{~cm}$ above the cotyledons by wounding the stem with a dissection needle ( $1.5 \mathrm{~mm}$ deep) and placing a square piece of agar inoculum $(1 \mathrm{~cm} \times 0.5 \mathrm{~cm} \times$ $2 \mathrm{~mm}$ ) on top of the wound. The inoculum was held in place by wrapping a $1-\mathrm{cm}$ piece of plastic soda straw (cut lengthwise) around the stem and the inoculum. A plastic collar (a Kim-Kap cap with the top cut off; Fisher Scientific, Pittsburgh, PA) was put on top of the tube and the inoculation site was covered with soil $(0.5 \mathrm{~cm}$ above top of soda straw). Parafilm was used to close the top of the plastic collar, leaving an opening of 2 to $4 \mathrm{~mm}$ around the stem. Inoculated plants were grown for 5 days in an illuminated incubator with a 16-h light / 8-h dark cycle at $27^{\circ} \mathrm{C}$. Inoculated plants were watered with $2 \mathrm{ml}$ immediately after inoculation and with $2 \mathrm{ml} 2$ days after inoculation. Lesion length on the stem was measured 5 days after inoculation. Lesions were only measured if the inoculum was still correctly placed on top of the wound. The individual who measured lesion length was blinded to the identity of each isolate. The highly virulent isolate 230-30-6 was included in each assay to verify that conditions allowed for successful infections.

For the pot assay, chickpea seeds were surface sterilized as described above, distributed on top of $2 \mathrm{~cm}$ of wet autoclaved soil in a metal tray $(53 \times 32 \times 6.4 \mathrm{~cm})$, covered with aluminum foil, and incubated at $24^{\circ} \mathrm{C}$ for 3 days. Conidia for inoculations were picked with a wooden stick from V8 medium plates ( 2 to 3 weeks old) and suspended in sterile $\mathrm{H}_{2} \mathrm{O}$. Germinated plants (2- to $3-\mathrm{cm}$ root) were inoculated $1 \mathrm{~cm}$ below the cotyledons by wounding the hypocotyl with a dissection needle $(1.5 \mathrm{~mm}$ deep) and injecting 1 to $2 \mu \mathrm{l}$ of water containing $10^{6}$ conidia into the wound. Fifteen germlings inoculated with the same isolate were planted in three rows of five plants each in polypropylene pans $(35.8 \times 30.8 \times 14.3 \mathrm{~cm})$ containing $10 \mathrm{~cm}$ of autoclaved soil. Cotyledons were covered with $1 \mathrm{~cm}$ of autoclaved soil, and incubated in a 16-h light / 8$\mathrm{h}$ dark cycle at $27^{\circ} \mathrm{C}$ for 3 weeks. Pots were watered as needed to keep the soil moist. Uninfected plants were treated like infected plants except water with no conidia was injected. Plant height was measured from cotyledons to the apex of each plant. General appearance of plants was also recorded at the end of the experiment.

\section{Induction of dispensable chromosome loss during vegetative growth.}

Loss of the dispensable chromosome in isolate $230 \mathrm{KO}-2$ was induced by exposure to benomyl as described in VanEtten et al. (in press).

\section{Statistical analysis.}

Data were analyzed with the Student's $t$ test. The effect size (d) also was calculated (Cohen 1969). Cohen's $d=\left\{\left(\operatorname{mean}_{1}-\right.\right.$ $\operatorname{mean}_{2}$ ) / standard deviation of unmanipulated population\}, except when calculating $d$ for pot assays when $d=\left\{\left(\right.\right.$ mean $_{1}-$ mean $_{2}$ ) / pooled standard deviation $\}$ (Cohen 1969). Effect size is interpreted as follows: $d>0.8$ is large; $d$ around 0.5 is moderate; and $d<0.2$ is small. We are using these statistical guideposts, which were recommended for behavioral research (Cohen 1969), because we are unaware of equivalent statistical guideposts for genetic research. The rationale for reporting the effect size is to provide a measure of the magnitude of the effect of the experimental manipulation, and to avoid reliance on either an arbitrary $P$ value such as $P<0.05$ to judge statistical significance, or percent change to quantify the outcome. The traditional $P$ value approach is potentially misleading because large differences can be declared nonsignificant based on large variances and a small $n$, while small effects can be declared significant based on small variance and a large $n$. In contrast to the traditional binomial decision of significant or nonsignificant, the effect size offers the advantage of being able to make standardized comparisons to past and future research. Percentages are also potentially misleading because the magnitude of the percent change depends on the absolute value of the control, or reference, data point. For example, given a control data point of $2 \mathrm{~mm}$, a $10 \%$ change in lesion length would represent an absolute change of only $0.2 \mathrm{~mm}$, but given a control data point of 20 $\mathrm{mm}$, a $10 \%$ change in lesion length would represent an absolute change of $2.0 \mathrm{~mm}$.

\section{ACKNOWLEDGMENTS}

We thank Catherine C. Wasmann and Hans D. VanEtten for providing the hygromycin $\mathrm{B}$ resistance cassette (pCWHyg1). We also thank Patrick J. O'Connor for help with statistical analyses, Scott Gold for critical reading of the manuscript, and Patton Hilliard for technical assistance. The work described in this paper was supported by the National Science Foundation under Grant No. MCB-9507263.

\section{LITERATURE CITED}

Bailey, J. A., and Mansfield, J. W., eds. 1982. Phytoalexins. Blackie and Son, Glasgow, Scotland.

Barz, W., and Welle, R. 1992. Biosynthesis and metabolism of isoflavones and pterocarpan phytoalexins in chickpea, soybean and phytopathogenic fungi. Pages 139-164 in: Phenolic Metabolism. H. A. Stafford and R. K. Ibrahim, eds. Plenum Press, New York.

Ciuffetti, L. M., and VanEtten, H. D. 1996. Virulence of a pisatin demethylase-deficient Nectria haematococca MPVI isolate is increased by transformation with a pisatin demethylase gene. Mol. PlantMicrobe Interact. 9:787-792.

Cohen, J. 1969. Statistical Power Analysis for Behavioral Sciences. Academic Press, New York.

Covert, S. F., Enkerli, J., Miao, V. P. W., and VanEtten, H. D. 1996. A gene for maackiain detoxification from a dispensable chromosome of Nectria haematococca. Mol. Gen. Genet. 251:397-406.

Ebel, J. 1986. Phytoalexin synthesis: The biochemical analysis of the induction process. Annu. Rev. Phytopathol. 24:235-264.

Enkerli, J. 1997. Functional and structural analysis of a dispensable chromosome in Nectria haematococca. Ph.D. dissert. University of Georgia, Athens.

Enkerli, J., Bhatt, G., and Covert, S. F. 1997. Nht1, a transposable element cloned from a dispensable chromosome in Nectria haematococca. Mol. Plant-Microbe Interact. 10:742-749.

Hain, R., Reif, H.-J., Krause, E., Langebartels, R., Kindl, H., Vornam, B., Wiese, W., Schmelzer, E., Schreier, P. H., Stöcker, R. H., and Stenzel, K. 1993. Disease resistance results from foreign phytoalexin expression in a novel plant. Science 361:153-156.

Kistler, H. C., Meinhardt, L. W., and Benny, U. 1996. Mutants of Nec- 
tria haematococca created by a site-directed chromosome breakage are greatly reduced in virulence toward pea. Mol. Plant-Microbe Interact. 9:804-809.

Lucy, M., Mathews, P., and VanEtten, H. D. 1988. Metabolic detoxification of the phytoalexins maackiain and medicarpin by Nectria haematococca field isolates: Relationship to virulence on chickpea. Physiol. Mol. Plant Pathol. 33:187-199.

Miao, V. P., Covert, S. F., and VanEtten, H. D. 1991. A fungal gene for antibiotic resistance on a dispensable ("B") chromosome. Science 254:1773-1776.

Miao, V. P. W., and VanEtten, H. D. 1992a. Three genes for metabolism of the phytoalexin maackiain in the plant pathogen Nectria haematococca: Meiotic instability and relationship to a new gene for pisatin demethylase. Appl. Environ. Microbiol. 58:801-808.

Miao, V. P. W., and VanEtten, H. D. 1992b. Genetic analysis of the role of phytoalexin detoxification in virulence of the fungus Nectria haematococca on chickpea (Cicer arietinum). Appl. Environ. Microbiol. 58:809-814.

Miller, P. M. 1955. V-8 juice agar as a general-purpose medium for fungi and bacteria. Phytopathology 45:461-463.

Müller, K. O., and Börger, H. 1940. Experimentelle Untersuchungen über die Phytophthora - Resistenz der Kartoffel. Arb. Biol. Reichsanst. Land. Forstwirtsch. Berlin-Dahlem. 23:189-231.

Reimmann, C., and VanEtten, H. D. 1994. Cloning and characterization of the PDA6-1 gene encoding a fungal cytochrome P-450 which detoxifies the phytoalexin pisatin from garden pea. Gene 146:221-226.

Sambrook, J., Fritsch, E. F., and Maniatis, T. A. 1989. Molecular Cloning: A Laboratory Manual. 2nd ed. Cold Spring Harbor Laboratory, Cold Spring Harbor, NY.

Schäfer, W., Straney, D., Ciuffetti, L., VanEtten, H. D., and Yoder, O. C. 1989. One enzyme makes a fungal pathogen, but not a saprophyte, virulent on a new host plant. Science 246:247-249.

Sharma, R. P., and Salunkhe, D. K., eds. 1991. Mycotoxins and Phytoalexins. CRC Press, Boston.

Stevens, R. B., ed. 1974. Mycology Guidebook. University of Washington Press, Seattle.

VanEtten, H. D. 1973. Differential sensitivity of fungi to pisatin and to phaseollin. Phytopathology 63:1477-1482

VanEtten, H. D., Funnell-Baerg, D., Wasmann, C., and McCluskey, K. 1994. Location of pathogenicity genes on dispensable chromosomes in Nectria haematococca MP VI. Antonie Van Leeuwenhoek 65:263267.

VanEtten, H. D., Jorgensen, S., Enkerli, J., and Covert, S. F. Inducing the loss of conditionally dispensable chromosomes in Nectria haematococca during vegetative growth. Curr. Genet. (In press.)

VanEtten, H. D., Matthews, D. E., Matthews, P. S., Miao, V., Maloney, A., and Straney, D. 1989. A family of genes for phytoalexin detoxification in the plant pathogen Nectria haematococca. Pages 219-228 in: Signal Molecules in Plants and Plant-Microbe Interactions. B. Lugtenberg, ed. Springer-Verlag, Berlin.

Wasmann, C. C., and VanEtten, H. D. 1996. Transformation-mediated chromosome loss and disruption of a gene for pisatin demethylase decrease the virulence of Nectria haematococca on pea. Mol. PlantMicrobe Interact. 9:793-803.

Weltring, K.-M. 1992. Phytoalexins in the relation between plants and their fungal pathogens. Pages 111-124 in: Molecular Biology of Filamentous Fungi. U. Stahl and P. Tudzynski, eds. VCH, Weinheim, Germany.

Weltring, K.-M., Turgeon, B. G., Yoder, O. C., and VanEtten, H. D. 1988. Isolation of a phytoalexin-detoxification gene from the plant pathogenic fungus Nectria haematococca by detecting its expression in Aspergillus nidulans. Gene 68:335-344. 